\title{
Evolución a lo largo del ciclo de puesta del contenido de ácidos grasos omega-3 en huevos enriquecidos de producción ecológica
}

\author{
M. Tor ${ }^{1, *}$, M. Llovera2 ${ }^{2}$ J.A. Moreno ${ }^{1}$ y E. Angulo ${ }^{1}$ \\ 1 Departament de Producció Animal. Universitat de Lleida. Av. Rovira Roure 191. 25198 Lleida \\ 2 Serveis Científico-Tècnics. Universitat de Lleida
}

\section{Resumen}

En este trabajo se estudia la puesta de gallinas en producción ecológica alimentadas con pienso enriquecido con un $4 \%$ de harina de pescado y $2,95 \%$ de aceite de soja hasta las 90 semanas de vida. Se caracteriza la calidad del huevo y se estudia la evolución de la composición de su grasa. Simultáneamente se evalúa la posibilidad de simplificar el método analítico para la composición de la grasa, omitiendo el paso de extracción de la grasa, previa a la transesterificación. Las características de los huevos estudiados se corresponden con los valores bibliográficos de producción ecológica, presentando un peso medio de $57,25 \pm 0,41$ g, un índice de forma de 80,42 $\pm 0,43$, un grosor de cáscara de 379,33 $\pm 4,56 \mu \mathrm{m}$, un valor de unidades Haugh de $80,17 \pm 0,88$ y un color en la escala DSM de entre 2 y 3 . Entre la semana 1 y la semana 70 de puesta, se observa un incremento cercano al 2,2\% en el porcentaje de ácidos grasos polinsaturados y un decremento equivalente en el de los monoinsaturados. El contenido de ácidos grasos $\omega-3$ aumenta de un $2,89 \pm 0,06 \%$ al inicio de la puesta, hasta un $3,2 \pm 0,06 \%$ a las 70 semanas. Con respecto al método de determinación de la grasa, la transesterificación directa de la grasa sobreestimó el contenido de ácidos grasos saturados y subestimó los monoinsaturados respecto al método convencional, en un $1 \%$. Esta diferencia, aunque significativa, en un contexto de controles de rutina, no reviste relevancia. El contenido de ácidos grasos polinsaturados, $\omega-3$ incluidos, no se ve afectado por el método analítico.

Palabras clave: Huevo, ácidos grasos, ácido docosahexaenoico, ácido eicosapentaenoico, transesterificación.

\section{Abstract \\ Evolution of the omega-3 fatty acid content in enriched organic eggs throughout the laying period}

The evolution of the fat quality of the eggs from organic production was studied. Hens were fed a diet enriched with $4 \%$ fish meal and $2.95 \%$ soy oil during the laying period, until 90 weeks of age. Simultaneously the possibility of simplifying the fat analysis method was evaluated by omitting the step of extraction prior to transesterification of the fat. The egg quality values obtained agree with the literature on organic production, having an average weight of $57.25 \pm 0.41 \mathrm{~g}$, a shape rate of $80.42 \pm 0.43$, a shell thickness of $379.33 \pm 4.56$ microns, a Haugh value of $80.17 \pm 0.88$ units and a DSM colour score of between 2 and 3. Between 1 and 70 lay weeks, an increase of about $2.2 \%$ in the percentage of polyunsaturated fatty acids was observed and a concomitant decrease in monounsaturated fatty acid percent-

* Autor para correspondencia: mtor@prodan.udl.cat http://dx.doi.org/10.12706/itea.2015.010 
age. The content of $\omega-3$ fatty acids increased from $0.06 \pm 2.89 \%$ at the start of laying period to $3.2 \pm 0.06 \%$ at 70 weeks. Regarding the fat analysis method, the direct transesterification of the fat led to an overestimated content of saturated fatty acids and an underestimated content of monounsaturated fatty acid. This difference of $1 \%$, although significant, is of no relevance within the context of routine checks. Any polyunsaturated fatty acid, $\omega-3$ content included, was not affected by the analytical method.

Key words: Egg, fat composition, docosahexaenoic fatty acid, eicosapentaenoic fatty acid, transesterification.

\section{Introducción}

La producción ganadera ecológica está estrictamente regulada y únicamente permite el uso de materias primas de producción ecológica, evitando el uso de sustancias sintéticas. Al mismo tiempo pone especial énfasis en el bienestar animal, tanto en a las densidades de alojamiento como al acceso a espacios exteriores que deben tener los animales. El consumo de alimentos ecológicos está en expansión, sus consumidores les atribuyen unas mejores características nutricionales y organolépticas y los valoran como una buena vía para proteger el medio ambiente (Kouba, 2003). Sin embargo no siempre existen evidencias científicas suficientes que sustenten esta percepción.

Por otro lado, uno de los aspectos más negativos de los cambios en los hábitos alimentarios contemporáneos, ha sido el incremento en el consumo de grasas polinsaturadas con alto contenido en ácidos grasos $\omega-6$, en detrimento de las que contienen $\omega-3$. En la dieta occidental actual, el ratio $\omega-6 / \omega-3$ se sitúa en valores de 10-20/1 en contraposición a valores cercanos a $1 / 1$ que tenían las dietas de nuestros ancestros (Molendi-Coste et al., 2011). Existen numerosas recomendaciones en el sentido de disminuir dicho ratio en la dieta y las estrategias más inmediatas consisten en incluir alimentos ricos en grasas $\omega-3$. EI pescado o la semilla de lino, ricos en ácidos grasos $\omega$-3 de cadena larga y ácido $\alpha$-linolénico respectivamente, son dos alimentos candidatos frecuentes a incluir en la dieta, aun- que presentan algunos inconvenientes, como son el alto contenido en metales pesados de algunos pescados o la presencia de factores antinutritivos de la semilla de lino (Carraro et al., 2012). Por todo ello, no siempre se consigue alcanzar el nivel recomendado de inclusión en la dieta. En este contexto, se ha incrementado el interés comercial por producir alimentos enriquecidos con ácidos grasos de cadena larga y esta quizás sea una buena solución, a largo plazo, para solventar el déficit crónico en ácidos grasos $\omega$-3 de las dietas occidentales (Harris, 2007).

El huevo de gallina enriquecido en ácidos grasos $\omega$-3 se comercializa actualmente con regularidad. Tiene la ventaja de ser un alimento ampliamente aceptado, que no suele tener restricciones de naturaleza social o religiosa, cuyo contenido de ácidos grasos polinsaturados puede ser modificado por la dieta (Fraeye et al., 2012). Se ha podido establecer una relación directa entre la composición de la grasa de la dieta y la composición de la grasa del huevo, de forma que el productor puede responder a la demanda del mercado o a nuevas directrices nutricionales (Baucells et al., 2000). Aun así, el tiempo necesario para la estabilización de los niveles de ácidos grasos $\omega$-3 en el huevo puede ser variable (Petrovic et al., 2012) y por otro lado, muchos de los estudios realizados (Baucells et al., 2000; Carvalho et al., 2009; Petrovic et al., 2012) no abarcan el ciclo de puesta completo, sino que se han realizado antes de la quincuagésima semana de puesta. Por ello, parece interesante profundizar en la deposición 
de los ácidos grasos $\omega$-3 especialmente en los periodos inicial y final de la puesta.

En este trabajo se hace un seguimiento, a lo largo de un ciclo de puesta completo de 70 semanas, de la composición de la grasa del huevo en gallinas de producción ecológica con una alimentación enriquecida en ácidos grasos $\omega-3$, a través de la incorporación de harina de pescado y aceite de soja en su dieta. Se pretende conseguir que los huevos alcancen el nivel mínimo establecido para la indicación "alto contenido en ácido docosahexaenoico (DHA)" (186 mg/100 g y $64 \mathrm{mg} / 100 \mathrm{kcal}$ ) cumpliendo los Reglamentos (UE) 116/ 2010 y 432/2012. En segundo lugar se evalúa el efecto de la transesterificación directa sobre la matriz, con el objetivo de simplificar la metodología de análisis de la composición de la grasa y facilitar así los controles analíticos de rutina.

\section{Material y métodos}

Este trabajo ha sido realizado en una granja ecológica comercial controlada por el Servicio de Certificación CAAE de Andalucía y Castilla La Mancha (ES-AN-00-AE). La normativa de producción ecológica asegura una densidad máxima en la zona cubierta de 6 gallinas $/ \mathrm{m}^{2}$ con acceso a zonas de aire libre de 4 $\mathrm{m}^{2}$ por gallina, durante al menos $1 / 3$ de su vida. La luz natural puede ser complementada hasta un máximo de 16 horas con un periodo de descanso nocturno continuo de al menos 8 horas. La zona cubierta de la explotación disponía de 2/3 partes de aseladero y el resto de la superficie cubierta con viruta de madera. Las naves estaban provistas de un nidal comunal longitudinal central escamoteable. Se usaron comederos circulares $(4 \mathrm{~cm} /$ gallina alojada) y bebederos de tetina con recuperador (1/10 gallinas alojadas).

La explotación controlada alojaba 3.000 gaIlinas Lohmann Brown con un ciclo de puesta largo, entre las 20 y 90 semanas de vida. El pienso y el agua se suministraron ad libitum. Se inició el suministro del pienso de puesta la semana 18 de vida y su formula porcentual se describe en la Tabla 1. Puesto que se suministró una dieta enriquecida en ácidos grasos $\omega-3$, en la Tabla 2 se detalla la composición analítica del pienso, incluyendo el perfil de ácidos grasos, así como él de la harina de pescado utilizada en su formulación.

En la primera semana, a las 50 y a las 70 semanas de puesta se tomaron sendas muestras de 15 huevos elegidos aleatoriamente. Paralelamente, en la semana 20, se tomó una muestra adicional de 24 huevos para determinar las características generales de calidad del huevo. Sobre el huevo entero se determinó el peso, el índice de forma (IF) (relación porcentual de la máxima anchura respecto la distancia entre los dos polos del huevo) y los porcentajes en peso de cáscara, clara y yema. Sobre la cáscara se determinó el grosor mediante un micrómetro específico 4001-DIG (Baxlo Precisión, Polinya, España), con una sensibilidad de $1 \mu \mathrm{m}$ y provisto de contactos para superficies curvas. El color de la cáscara y el de la yema, se determinaron obteniendo las coordenadas $L^{*}, a^{*}, b$ * en el espacio CIELAB mediante un colorímetro Konica Minolta CM-700d (Konica Minolta Sensing Inc., Osaka, Japón) con un diámetro de máscara de 8 $\mathrm{mm}$, iluminante D65 y ángulo de observación de $10^{\circ}$. En el caso de la yema se utilizó como contenedor una cápsula de vidrio óptico cilíndrica de $34 \mathrm{~mm}$ de diametro (Hellma, Müllheim, Alemania). Se realizó una segunda determinación del color de la yema mediante el abanico colorimétrico de DSM (DSM Egg Yolk Color Fan, Heerlen, Holanda). Se determinó la consistencia de la clara obteniendo el valor de unidades Haugh (Haugh, 1937), midiendo la altura del albumen mediante un micrómetro Haugh (Baxlo Precisión, Polinya, España) con una sensibilidad de 0,1 $\mathrm{mm}$. El mismo instrumento se utilizó para medir la 
Tabla 1. Fórmula porcentual del pienso*

Table 1. Formula of the concentrate feed used during the laying period

\begin{tabular}{lr}
\hline Ingredientes & $\%$ \\
\hline Cebada 6 carreras & 20,00 \\
Trigo blando & 38,88 \\
Soja $37-40 \%$ & 23,64 \\
Harina de pescado 60/9/20 & 4,00 \\
Aceite de soja & 2,95 \\
DL Metionina & 0,19 \\
L-Lisina HCl & 0,09 \\
Covyzyme 50 (1) & 0,15 \\
Covimix gallinas $10 \% \mathrm{ECO}^{(2)}$ & 10,00 \\
Covitsa Calcio Forte & $(3)$ \\
\hline
\end{tabular}

*Pienso fabricado bajo el amparo de la normativa de producción ecológica. (1), (2) y (3), según marcas registradas y comercializadas por COVITSA, Lleida, España.

(1) Premezcla E-1604 enzimas: endo-1,4 $\beta$-xilanasa, EC 3.2.1.8 $70 \mathrm{U} / \mathrm{g}$ y endo-1,3(4)- $\beta$-Glucanasa, EC 3.2.1.6 $100 \mathrm{U} / \mathrm{g}$.

(2) Por kg de pienso: vitamina A, $12.000 \mathrm{UI}$; vitamina D3, $2.000 \mathrm{Ul}$; vitamina $\mathrm{E}, 20 \mathrm{mg}$; vitamina $\mathrm{K} 3,1 \mathrm{mg}$; vitamina $\mathrm{B} 2,3 \mathrm{mg}$; vitamina $\mathrm{B} 6,1 \mathrm{mg}$; vitamina B12, 0,020 mg; ácido pantoténico, 10 mg; ácido nicotínico, 25 mg; cloruro de colina, $300 \mathrm{mg}$; hierro, $30 \mathrm{mg}$; yodo, 0,81 mg; cobre, $4 \mathrm{mg}$; manganeso, $70 \mathrm{mg}$, zinc, $30 \mathrm{mg}$; selenio, 0,10 mg; cloruro de sodio, 3,45 g; fosfato monocálcico defluorado, 8,62 $\mathrm{g}$.

(3) Por kg de pienso: vitamina D3, $1.000 \mathrm{Ul}$; vitamina C, 0,30 Ul; cloruro de colina, $75 \mathrm{mg}$; manganeso, $12,55 \mathrm{mg}$; zinc, $17,80 \mathrm{mg}$; selenio, 0,04 ; cobre, $1,5 \mathrm{mg}$; hierro, $1,75 \mathrm{mg}$, oxido de magnesio, $12,5 \mathrm{mg}$.

altura de la yema y calcular el índice de yema (expresado como la relación porcentual entre la altura y el diámetro de la yema). El contenido de materia seca de la clara y de la yema se determinó por secado de la muestra a $100-102^{\circ} \mathrm{C}$ en estufa de aire forzado durante 24 horas. El contenido de proteína se determinó por el método Kjeldahl (AOAC, 2000) y el contenido de lípidos totales por el método Soxhlet (AOAC, 2000). El contenido de energía bruta se determino mediante una autobomba calorimétrica (Gallenkamp Autobomb CBA 305, Londres, Reino Unido).

Los análisis de composición de la grasa se realizaron con y sin extracción previa de la misma, ambos por triplicado. La extracción de los lípidos en frío se realizó mediante $50 \mathrm{~mL}$ de una mezcla de triclorometano/metanol, $1 / 1, v / v$, sobre una muestra de yema liofilizada de 1g. La transesterificación de los ácidos gra- 
Tabla 2. Composición analítica de pienso y materias primas Table 2. Analytical composition of the raw material and fish meal

\begin{tabular}{|c|c|c|}
\hline & Pienso enriquecido & Harina de pescado \\
\hline Materia seca (\%) & 91,60 & 92,33 \\
\hline Proteína bruta (\%) & 17,58 & 66,36 \\
\hline Extracto etéreo (\%) & 10,46 & 12,14 \\
\hline \multicolumn{3}{|l|}{ Composición de ácidos grasos (\%) } \\
\hline C14:0 & 1,38 & 5,48 \\
\hline C14:1, cis-9 & 0,04 & 0,14 \\
\hline C16:0 & 19,48 & 20,66 \\
\hline C16:1, cis-9 & 2,17 & 6,03 \\
\hline C17:0 & 0,38 & 1,36 \\
\hline C18:0 & 9,28 & 6,05 \\
\hline C18:1, cis-9 & 39,46 & 31,81 \\
\hline C18:2,cis-9, 12 & 22,57 & 4,45 \\
\hline C18:3,cis-9, 12,15 & 2,19 & 1,45 \\
\hline C20:0 & 0,17 & 0,34 \\
\hline C20:1, cis-11 & 1,16 & 5,35 \\
\hline C20:2, cis-11,14 & 0,57 & 0,74 \\
\hline C20:4,cis-5,8,11,14 & 0,20 & 0,63 \\
\hline C20:5,cis-5,8,11,14,17 & 0,00 & 3,30 \\
\hline C22:1,cis-13 & 0,14 & 0,86 \\
\hline C22:6, cis- $4,7,10,13,16,19$ & 0,68 & 10,05 \\
\hline C24:0 & 0,06 & 0,14 \\
\hline C24:1,cis-15 & 0,08 & 1,16 \\
\hline Ácidos grasos saturados & 30,75 & 34,03 \\
\hline Ácidos grasos monoinsaturados & 43,05 & 45,35 \\
\hline Ácidos grasos poliinsaturados & 26,21 & 20,62 \\
\hline Ácidos grasos poliinsaturados $\omega-6$ & 23,34 & 5,82 \\
\hline Ácidos grasos poliinsaturados $\omega-3$ & 2,87 & 14,80 \\
\hline
\end{tabular}


sos, directamente sobre la yema o de la grasa previamente extraída, se realizó mediante 1 $\mathrm{mL}$ de una solución de trifluoruro de boro $\left(\mathrm{BF}_{3}\right)$ en metanol al $20 \%$ sobre un peso de muestra equivalente a un contenido de unos $25 \mathrm{mg}$ de grasa a $80^{\circ} \mathrm{C}$ durante dos horas. La recuperación de los esteres metílicos se llevó a cabo mediante dos lavados con $1 \mathrm{~mL}$ de hexano. Como patrón interno se utilizó $1 \mathrm{mg}$ de 1,2,3-Tripentadecanoil-glicerol. El análisis de los ésteres metílicos de los ácidos grasos se realizó por cromatografía gas-líquido en columna capilar (SP2330; Supelco, Tres Cantos, Madrid, España) de $30 \mathrm{~m}$, un detector tipo FID, utilizando como gas portador helio a un flujo de $1 \mathrm{~mL}$ minuto (Muñoz et al., 2010). La identificación de los ácidos grasos se realizó por comparación de tiempos de retención respecto a un patrón externo (F.A.M.E. Mix, C4-C24, Sigma-Aldrich, St. Louis, EE.UU.).

Para el análisis estadístico de la composición de la grasa, se utilizó el del programa estadístico SAS (1996). Para la comparación de medias se utilizó el procedimiento MIXED, con un modelo que incluyó el método analítico y la semana de puesta como factores fijos y el huevo como factor aleatorio. Las correlaciones entre los dos métodos analíticos se determinaron mediante el procedimiento CORR.

\section{Resultados y discusión}

Calidad general del huevo

En la Tabla 3 se describen las características generales de calidad en el ciclo de puesta estudiado, tanto del huevo entero como de la cáscara, de la clara y de la yema. Existen estudios previos del efecto del sistema de cría ecológico sobre la calidad del huevo. Se ha descrito, en huevos ecológicos, una mayor consistencia del albumen, un color de yema menos intenso y una cascara más pesada que en huevos convencionales (Küçükyılmaz et al., 2012). Los va-
Tabla 3. Características generales de calidad del huevo en la vigésima semana de puesta (Media \pm error estándar)

Table 3. General characteristics of egg quality in the twentieth week of laying

\begin{tabular}{|c|c|}
\hline \multicolumn{2}{|l|}{ Huevo entero } \\
\hline Peso (g) & $57,27 \pm 0,41$ \\
\hline Índice de forma & $80,42 \pm 0,43$ \\
\hline Cáscara (\%) & $12,92 \pm 0,11$ \\
\hline Clara (\%) & $60,93 \pm 0,38$ \\
\hline Yema (\%) & $26,11 \pm 0,41$ \\
\hline \multicolumn{2}{|l|}{ Cáscara } \\
\hline Grosor $(\mu \mathrm{m})$ & $379,33 \pm 4,56$ \\
\hline \multicolumn{2}{|l|}{ Color } \\
\hline$L^{*}$ & $62,83 \pm 0,28$ \\
\hline$a *$ & $16,32 \pm 0,31$ \\
\hline$b^{*}$ & $26,54 \pm 0,50$ \\
\hline \multicolumn{2}{|l|}{ Clara } \\
\hline Consistencia (unidades Haugh) & $80,17 \pm 0,88$ \\
\hline Materia seca (\%) & $23,81 \pm 4,20$ \\
\hline \multicolumn{2}{|l|}{ Yema } \\
\hline Índice de yema & $43,61 \pm 0,72$ \\
\hline \multicolumn{2}{|l|}{ Color } \\
\hline escala DSM & $2,58 \pm 0,09$ \\
\hline$L^{*}$ & $58,03 \pm 0,28$ \\
\hline$a *$ & $-1,06 \pm 0,06$ \\
\hline$b *$ & $22,32 \pm 0,32$ \\
\hline Materia seca (\%) & $47,58 \pm 1,13$ \\
\hline
\end{tabular}

lores de calidad obtenidos en el presente estudio, están dentro de los estándares de calidad aceptables, aunque son de destacar algunas de sus características. El peso del huevo es relativamente bajo (Tabla 3), situándose el 
valor medio en la categoría comercial $\mathrm{M}$ y con un índice de forma alto, superior a 80 . Aunque Clerici et al. (2006), no encuentran diferencias en el índice de forma entre huevos ecológicos y convencionales, nuestros resultados estarían más de acuerdo con Küçükyılmaz et al. (2012), quienes describen valores altos de índice de forma en huevos ecológicos y en consecuencia previsiblemente más resistentes a la rotura (Altuntas y Sekeroglu, 2008). Se ha descrito en varios estudios que los huevos ecológicos tienen cáscaras más gruesas que los convencionales (Rizzi et al., 2006; Mugnai et al., 2009; Küçükyılmaz et al., 2012). Los datos de grosor y porcentaje de cáscara se presentan en la Tabla 3.

El porcentaje de yema ha alcanzado unos valores frecuentes en huevo ecológico, mientras que el porcentaje de clara, que no llega al $61 \%$ se puede considerar relativamente bajo. Por el contrario la estructura de la clara es consistente, con un valor superior a las 80 unidades Haugh. Finalmente cabe destacar el color de la yema extremadamente pálido, con valores entre 2 y 3 en la escala DSM, destacando los valores negativos de rojo (Tabla 3 ).

\section{Evolución de la composición de la grasa}

La evolución de la composición de la grasa se presenta en la Tabla 4 . No se han detectado variaciones a lo largo del ciclo de puesta en el contenido de ácidos grasos saturados, con valores situados alrededor del $32 \%$. Por el contrario, sí hay variaciones significativas entre el inicio de la puesta y los dos controles posteriores a 50 y 70 semanas en el caso de los insaturados. El contenido de monoinsaturados disminuye ligeramente, alrededor de un $2,2 \%$ mientras que los polinsaturados aumentan en la misma proporción. La disminución de los ácidos grasos monoinsaturados se explicaría en gran medida por cambios en el contenido de los ácidos grasos palmitoleico, oleico y $\alpha$-linoleico. El incremento en el contenido de ácidos grasos polinsaturados a lo largo del ciclo de puesta se detecta tanto en los $\omega$-6 como en los $\omega$-3. El contenido de ácidos grasos $\omega-3$ aumentó a medida que avanzaba el ciclo de puesta, pasando de $2,89 \%$ al inicio a 3,2\% al final de la misma (Tabla 4). En el caso concreto de los ácidos grasos saturados no se detectaron diferencias entre los tres momentos del ciclo de puesta estudiados.

Sobre el efecto del sistema productivo en la composición de la grasa de la yema de huevo, hay resultados dispares en la bibliografía, probablemente debido a que la composición depende más del tipo de dieta que el animal ingiere en realidad, que del sistema productivo propiamente dicho. $Y$ no siempre hay una relación directa entre ambos. En cría ecológica, cuando la superficie al aire libre suministra suficiente pasto a lo largo del ciclo de puesta, suele detectarse un aumento de los ácidos grasos $\omega$-3. Pero esto no siempre ocurre, porque aunque la superficie al aire libre sea amplia, la vegetación disponible puede no ser suficiente para que la composición de la grasa se vea afectada. Este sería el caso del presente trabajo, donde los altos niveles de ácidos grasos $\omega-3$ mantenidos durante todo el ciclo de puesta, son atribuibles al DHA procedente de la suplementación del pienso con harina de pescado y aceite de soja, más que al consumo de forraje verde, del cual hubo poca disponibilidad en la zona al aire libre. En realidad el contenido de ácido $\alpha$-linolénico detectado fue bajo $(<0,6 \%)$ mientras que el contenido de DHA supera el 2,3\% desde el inicio de la puesta, ya que se había iniciado el suministro de pienso enriquecido dos semanas antes.

Existen referencias previas de que los ácidos grasos de cadena larga (eicosapentaenoico (EPA) y DHA) se depositan y acumulan en la yema de huevo preferentemente en forma de DHA (Nitsan et al., 1999; Fredriksson et al., 2006; Bruneel et al., 2013). En la Tabla 4, se puede ver que más del $80 \%$ del contenido de ácidos grasos $\omega$-3 acumulados en el huevo 
Tabla 4. Evolución de la composición de la grasa de la yema a lo largo del ciclo de puesta (Media \pm error estándar)

Table 4. Evolution of the composition of the yolk fat during the laying period

\begin{tabular}{|c|c|c|c|}
\hline \multirow[b]{2}{*}{ Ácidos grasos (\%) } & \multicolumn{3}{|c|}{ Semana de puesta } \\
\hline & 1 & 50 & 70 \\
\hline C14:0 & $0,35 \pm 0,01^{b}$ & $0,41 \pm 0,01^{a}$ & $0,41 \pm 0,01^{a}$ \\
\hline C14:1,cis-9 & $0,06 \pm 0,003^{a}$ & $0,03 \pm 0,003^{b}$ & $0,05 \pm 0,003^{a}$ \\
\hline C16:0 & $23,43 \pm 0,20$ & $23,24 \pm 0,20$ & $23,84 \pm 0,21$ \\
\hline C16:1, cis-9 & $2,49 \pm 0,15^{a}$ & $1,90 \pm 0,15^{b}$ & $1,21 \pm 0,15^{c}$ \\
\hline C18:0 & $8,44 \pm 0,11^{a}$ & $8,32 \pm 0,11 a b$ & $8,05 \pm 0,11^{b}$ \\
\hline C18:1, trans-9 & $0,07 \pm 0,01^{b}$ & $0,19 \pm 0,01^{a}$ & $0,03 \pm 0,01^{c}$ \\
\hline C18:1,cis-9 & $46,82 \pm 0,33^{a}$ & $44,94 \pm 0,33^{b}$ & $45,90 \pm 0,34 \mathrm{ab}$ \\
\hline C18:2, cis-9,12 & $13,16 \pm 0,23^{b}$ & $14,72 \pm 0,23{ }^{a}$ & $14,89 \pm 0,23^{a}$ \\
\hline C18:3,cis-6,9,12 & $0,07 \pm 0,003^{c}$ & $0,11 \pm 0,003^{a}$ & $0,08 \pm 0,002^{b}$ \\
\hline C18:3,cis-9,12,15 & $0,50 \pm 0,01^{b}$ & $0,55 \pm 0,01^{a}$ & $0,59 \pm 0,01^{a}$ \\
\hline C20:1,cis-11 & $0,31 \pm 0,01$ & $0,31 \pm 0,01$ & $0,33 \pm, 01$ \\
\hline C20:2, cis- 11,14 & $0,25 \pm 0,02$ & $0,20 \pm 0,021$ & $0,25 \pm 0,012$ \\
\hline C20:3, cis-11,14,17 & $0,12 \pm 0,003^{a}$ & $0,15 \pm 0,003^{b}$ & $0,14 \pm 0,003^{b}$ \\
\hline C20:4,cis-5,8,11,14 & $1,24 \pm 0,045^{a}$ & $2,05 \pm 0,045^{b}$ & $1,24 \pm 0,046^{a}$ \\
\hline C20:5,cis-5,8,11,14,17 & & $0,006 \pm 0,002$ & \\
\hline C22:6, cis-4,7,10,13,16,19 & $2,39 \pm 0,063$ & $2,40 \pm 0,063$ & $2,61 \pm 0,064$ \\
\hline Ácidos grasos saturados & $32,22 \pm 0,24$ & $31,98 \pm 0,24$ & $32,30 \pm 0,24$ \\
\hline Ácidos grasos monoinsaturados & $49,75 \pm 0,28^{a}$ & $47,40 \pm 0,28^{b}$ & $47,53 \pm 0,28^{b}$ \\
\hline Ácidos grasos poliinsaturados & $17,73 \pm 0,26^{b}$ & $20,20 \pm 0,26{ }^{a}$ & $19,81 \pm 0,27^{a}$ \\
\hline Ácidos grasos poliinsaturados $\omega-6$ & $14,65 \pm 0,23 b$ & $16,97 \pm 0,23{ }^{a}$ & $16,39 \pm 0,23{ }^{a}$ \\
\hline Ácidos grasos poliinsaturados $\omega-3$ & $2,89 \pm 0,06^{b}$ & $2,97 \pm 0,06^{b}$ & $3,2 \pm 0,07^{a}$ \\
\hline
\end{tabular}

Para cada fila, valores seguidos de distinta letra en superíndice son diferentes $(P \leq 0,05)$.

efectivamente corresponden a DHA. Aunque en este caso se deba más a la naturaleza de la grasa añadida (su contenido en DHA supera el $10 \%$, Tabla 2), que a la transformación endógena de EPA en DHA, puesto que el contenido de EPA de la grasa añadida es bajo y en el pienso enriquecido no llegó a detectarse. En la yema de huevo, únicamente en la semana de puesta 50 se detectan trazas de EPA. Desde la perspectiva de la calidad nutricional, una proporción alta de DHA respecto al total de ácidos grasos $\omega$-3 supone una ven- 
taja, puesto que en humanos la transformación de EPA en DHA es especialmente pobre (Burdge, 2004) y el compuesto bioactivo con efectos beneficiosos sobre el cerebro y el sistema cardivascular es el DHA (Lagarde, 2008). Los Reglamentos (UE) 116/2010 y 432/2012 especifican que para poder etiquetar un producto alto DHA debe contener más de $\mathbf{4 0}$ $\mathrm{mg}$ por $100 \mathrm{~g}$ de producto y $100 \mathrm{kcal}$ y que la ingesta diaria para conseguir el efecto beneficioso es de $250 \mathrm{mg}$. Por tanto los huevos estudiados alcanzaron el nivel mínimo establecido para la indicación "alto contenido en DHA" (186 mg/100 g y $64 \mathrm{mg} / 100 \mathrm{kcal}$; Energía bruta clara y yema: 5.222 y 7.525 kcal respectivamente; Proteína bruta clara y yema: 87,32 y $32,7 \%$ respectivamente; Extracto etéreo yema: $54,58 \%$ ). Para conseguir el efecto beneficioso, sería necesaria una ingesta mínima diaria de $134 \mathrm{~g}$ (dos huevos de tamaño XL o tres de tamaño M). Probablemente esta ingesta supera largamente la mayoría de las recomendaciones nutricionales existentes, avaladas por el hecho de que existe una respuesta positiva dosis-dependiente entre el consumo de huevos y el riesgo de enfermedades cardiovasculares y diabetes (Li et al., 2013). Una ingesta de un huevo de tamaño medio diario supondría un aporte de $106 \mathrm{~g}$ de ácidos grasos $\omega$-3, equivalente al $42 \%$ de la dosis mínima para conseguir un efecto beneficioso.

Efecto del método analítico sobre la composición de la grasa

En cuanto al efecto del método de análisis sobre la composición de la grasa, cabe destacar que la transesterificación directa mediante $\mathrm{BF}_{3^{\prime}}$ en nuestras condiciones, ha sobreestimado el porcentaje de ácidos grasos saturados y ha subestimado el contenido de los monoinsaturados, en comparación a la extracción previa de la grasa mediante cloroformo/metanol (Tabla 5). Desde un punto de vista cuantitativo las diferencias más relevantes se centran en los áci- dos grasos saturados palmítico y esteárico y en los monoinsaturados palmitoleico y oleico. Sin embargo, no se ha detectado efecto del método analítico sobre el contenido de los ácidos grasos polinsaturados, siendo el coeficiente de correlación entre ambos métodos analíticos de $0,964(P<0,001)$ para el porcentaje total de los ácidos grasos polinsaturados; de 0,960 $(P<0,001)$ para el porcentaje de ácidos grasos $\omega-6$; de $0,920(P<0,001)$ para el porcentaje de ácidos grasos $\omega$-3 y de $0,742(\mathrm{P}<0,001)$ para el porcentaje de DHA.

La ventaja principal de realizar la transesterificación directa sobre la matriz, sin extracción previa de la grasa, es una mayor simplicidad analítica con el consiguiente ahorro de disolventes y tiempo, lo que redunda en una determinación más económica. Desde que Folch et al. (1957) propusieron su método de extracción de los lípidos en frío, han sido numerosas las propuestas para obviar este paso previo a la transesterificación, en multitud de matrices. En el caso de la yema de huevo, Wang et al. (2000) propusieron la transesterificacion directa mediante $\mathrm{HCl}$ como un método con resultados comparables a los métodos convencionales y con una repetibilidad incluso mejor. Por el contrario, Mazalli y Bragagnolo (2007) llegaron a la conclusión contraria y propusieron la extracción previa con cloroformo/metanol/agua seguida de una transesterificación con $\mathrm{BF}_{3}$ como el método óptimo. En el primer caso, la transesterificación directa, evita la fuente de error que puede suponer la extracción previa, y por tanto se alcanza una repetibilidad mayor. Por el contrario, la interferencia de la matriz en el proceso de transesterificación puede provocar un menor rendimiento del proceso, con el consiguiente sesgo de los valores.

En el presente trabajo se han utilizado un método con extracción previa y otro sin ella, en el marco de controles de rutina de huevos enriquecidos en ácidos grasos $\omega-3$. De acuerdo con la bibliografía, se han detectado algunas 
Tabla 5. Efecto del método de análisis ${ }^{1}$ sobre la composición de la grasa de la yema (Media \pm error estándar) Table 5. Effect of the method of analysis on the yolk fat composition

\begin{tabular}{lccl}
\hline & TD & E & P valor \\
\hline C14:0 & $0,40 \pm 0,01$ & $0,39 \pm 0,01$ & 0,050 \\
C14:1,cis-9 & $0,05 \pm 0,003$ & $0,05 \pm 0,003$ & 0,82 \\
C16:0 & $23,72 \pm 0,17$ & $23,28 \pm 0,16$ & 0,07 \\
C16:1,cis-9 & $1,41 \pm 0,12$ & $2,33 \pm 0,13$ & 0,001 \\
C18:0 & $8,45 \pm 0,09$ & $8,09 \pm 0,09$ & 0,006 \\
C18:1,trans-9 & $0,10 \pm 0,01$ & $0,08 \pm 0,01$ & 0,08 \\
C18:1,cis-9 & $45,98 \pm 0,27$ & $45,79 \pm 0,27$ & 0,046 \\
C18:2,cis-9,12 & $14,24 \pm 0,18$ & $14,27 \pm 0,19$ & 0,94 \\
C18:3,cis-6,9,12 & $0,08 \pm 0,002$ & $0,09 \pm 0,002$ & 0,002 \\
C18:3,cis-9,12,15 & $0,53 \pm 0,01$ & $0,56 \pm 0,01$ & 0,12 \\
C20:1,cis-11 & $0,30 \pm 0,01$ & $0,32 \pm 0,01$ & 0,03 \\
C20:2,cis-11,14 & $0,23 \pm 0,02$ & $0,24 \pm 0,02$ & 0,65 \\
C20:3,cis-11,14,17 & $0,14 \pm 0,002$ & $0,13 \pm 0,002$ & 0,29 \\
C20:4,cis-5,8,11,14 & $1,52 \pm 0,04$ & $1,50 \pm 0,04$ & 0,76 \\
C20:5,cis-5,8,11,14,17 & $0,001 \pm 0,001$ & $0,002 \pm 0,001$ & 0,79 \\
C22:6,cis-4,7,10,13,16,19 & $2,44 \pm 0,05$ & $2,49 \pm 0,05$ & 0,49 \\
Ácidos grasos saturados & $32,57 \pm 0,20$ & $31,77 \pm 0,20$ & 0,004 \\
Ácidos grasos monoinsaturados & $47,86 \pm 0,23$ & $48,59 \pm 0,23$ & 0,02 \\
Ácidos grasos poliinsaturados & $19,20 \pm 0,22$ & $19,30 \pm 0,22$ & 0,75 \\
Ácidos grasos poliinsaturados $\omega-6$ & $16,00 \pm 0,18$ & $16,01 \pm 0,19$ & 0,95 \\
Ácidos grasos poliinsaturados $\omega-3$ & $2,98 \pm 0,05$ & $3,05 \pm 0,05$ & 0,32 \\
\hline
\end{tabular}

${ }^{1}$ TD: transesterificación directa; E: método con extracción previa a la transesterificación.

diferencias entre ambos (Tabla 5). Aun así y teniendo en cuenta que el contenido de ácidos grasos saturados y monoinsaturados ha superado el $30 \%$ y el $45 \%$ respectivamente y que las diferencias entre métodos son menores al $1 \%$, puede considerarse que, aunque estas son significativas, no son relevantes a la hora de cuantificar el perfil completo de áci- dos grasos del huevo en este contexto. Únicamente en el caso del ácido palmitoleico la subestimación que supone la extracción directa puede considerarse relevante. Los valores del contenido de ácidos grasos $\omega$-3 no se han visto afectados por el método analítico, ni en su conjunto ni considerados de manera individual. 


\section{Bibliografía}

Altuntas E, Sekeroglu A (2008). Effect of egg shape index on mechanical properties of chicken eggs. Journal of Food Engineering 85: 606-612.

AOAC. 2000. Official Methods of Analysis, 17th ed. Assoc. Off. Anal. Chem., Gaithersburg, MD.

Baucells MD, Crespo N, Barroeta AC, López-Ferrer S, Grashorn MA (2000). Incorporation of different polyunsaturated fatty acids into eggs. Poultry Science 79: 51-59.

Bruneel C, Lemahieu C, Fraeye I, Ryckebosch E, Muylaert K, Buyse J, Foubert I (2013). Impact of microalgal feed supplementation on omega-3 fatty acid enrichment of hen eggs. Journal of Funcional Foods 5: 897-904.

Burdge G (2004). Alpha-linolenic acid metabolism in men and women: nutritional and biological implications. Current Opinion In Clinical Nutrition And Metabolic Care 7: 137-144.

Carraro JCC, Dantas MID, Espeschit ACR, Martino HSD, Ribeiro, SMR (2012). Flaxseed and human health: reviewing benefits and adverse effects. Food Reviews International 28: 203-230.

Carvalho PR, Pita MCG, Piber Neto E, Mendonca Junior CX (2009). Efficiency of PUFAs incorporation from marine sources in yolk egg's laying hens. Journal of Poultry Science 8: 603-614.

Clerici F, Casiraghi E, Hidalgo A, Rossi M (2006). Evaluation of eggshell quality characteristics in relation to the housing system of laying hens. Proceedings of the XII European Poultry Conference. 10-14 September 2006. Verona-Italia.

Folch J, Less M, Stanley GHS (1957). A simple method for the islolation and purification of total lipids from animal tissues. Journal of Biological Chemistry 226: 497-509.

Fraeye I, Bruneel C, Lemahieu C, Buyse J, Muylaert K, Foubert I (2012). Dietary enrichment of eggs with omega-3 fatty acids: A review. Food Research International 48: 961-969.

Fredriksson S, Elwinger K, Pickova J (2006). Fatty acid and carotenoid composition of egg yolk as an effect of microalgae addition to feed formula for laying hens. Food Chemistry 99: 530-537.
Haugh RR (1937). The Haugh unit for measuring egg quality. The US Egg and Poultry Magazine 43: 522-555, 572-573.

Harris WS (2007). International recommendations for consumption of long-chain omega-3 fatty acids. Journal of Cardiovascular Medicine 8: 50-52.

Kouba M (2003). Quality of organic animal products. Livestock Production Science 80: 33-40.

Küçükyılmaz K, Bozkurt M, Herken E, Çınar M, Çatlı AU, Binta E, Coven F (2012). Effects of rearing systems on performance, egg characteristics and immune response in two layer hen genotype. Asian-Australasian Journal of Animal Sciences 25: 559-568.

Lagarde M (2008). Docosahexaenoic acid: Nutrient and precursor of bioactive lipids. European Journal of Lipid Science and Technology 110: 673-678.

Li Y, Zhou C, Zhou X, Li L (2013). Egg consumption and risk of cardiovascular diseases and diabetes: a meta-analysis. Atherosclerosis 229: 524-530.

Mazalli M, Bragagnolo N, (2007). Validation of two methods for fatty acid analysis in eggs. Lipids 42: 483-490.

Molendi-Coste O, Legry V, Leclercq IA (2011). Why and how meet n-3 PUFA dietary recommendations? Gastroenterology Research and Practice, Volume 2011, Article ID 364040, 11 p.

Mugnai C, Dal Bosco A, Castellini C (2009). Effects of rearing system and season on the performance and egg charasteristics of Ancona laying hens. Italian Journal of Animal Science 8: 175-188.

Muñoz R, Vilaró F, Eras J, Estany J, Tor M (2010). Fast determination of oleic acid in pork by flow injection analysis/mass spectrometry. Rapid Communications in Mass Spectrometry 25: 1082-1088.

Nitsan Z, Mokady S, Sukenik A (1999). Enrichment of poultry products with omega-3 fatty acids bydietary supplementation with the alga Nannochloropsis and mantur oil. Journal of Agricultural and Food Chemistry 47: 5127-5132.

Petrovic M, Galic M, Karacic V, Gottstein Z, Mazija H, Medic H (2012). Enrichment of eggs in n-3pol- 
yunsaturated fatty acids by feeding hens with different amount of linseed oil in diet. Food chemistry 135: 1563-1568.

Rizzi L, Simioli G, Martelli G, Paganelli R, Sardi L (2006). Effects of organic farming on egg quality and welfare of laying hens. Proceedings of the XII European Poultry Conference. 10-14 September 2006. Verona-Italia.
SAS, SAS/STAT User's Guide: statistics, SAS Inst. Inc., Cary, North Caroline, 1996.

Wang $Y$, Sunwoo H, Gherian G, Sim JS (2000). Fatty acid determination in chicken egg yolk: a comparison of different methods. Poultry Science 79: 1168-1171.

(Aceptado para publicación el 6 de noviembre de 2014) 